\title{
A primer on harnessing non-enzymatic post- translational modifications for drug design
}

\author{
Marcus J. C. Long ${ }^{\ddagger}$, Phillippe Ly†, and Yimon Aye+* \\ †Swiss Federal Institute of Technology in Lausanne (EPFL), 1015 Lausanne, Switzerland \\ ‡University of Lausanne, 1015 Lausanne, Switzerland \\ "Correspondence: yimon.aye@epfl.ch
}




\section{Summary}

Of the manifold concepts in drug discovery and design, covalent drugs have re-emerged as one of the most promising over the past 20 -or so years. All such drugs harness the ability of a covalent bond to drive an interaction between a target biomolecule, typically a protein, and a small molecule. Formation of a covalent bond necessarily prolongs target engagement, opening avenues to targeting shallower binding sites, protein complexes, and other difficult to drug manifolds, amongst other virtues. This opinion piece discusses frameworks around which to develop covalent drugs. Our argument, based on results from our research program on natural electrophile signaling, is that targeting specific residues innately involved in native signaling programs are ideally poised to be targeted by covalent drugs. We outline ways to identify electrophile-sensing residues, and discuss how studying ramifications of innate signaling by endogenous molecules can provide a means to predict drug mechanism and function and assess on- versus off-target behaviors. 
Analysis of many modern covalent drugs shows that they consist of two distinct parts. One section, which is typically considerably larger than the other (upwards of $\sim 250$ Daltons), is a "ligandable" fragment. This piece binds to protein(s) non-covalently, inhibiting the activity of the target. The second portion is typically lower in molecular weight ( $\sim 60$ Daltons), but contains a weakly-reactive Michael acceptor. The combination of the twain forges a molecule that, ideally, binds the target in a rapidreversible manner, ushering formation of a covalent bond selectively to the target protein, yielding an irreversibly-bound complex. This choreography imparts enzyme-like rate acceleration to covalentbond formation, which in the absence of ligand binding, is (ideally) negligible: many acrylamidecontaining covalent inhibitors, for instance, have second-order rates of inhibition $>10^{5} \mathrm{M}^{-1} \mathrm{~s}^{-1}$ $\left[t_{1 / 2}\right.$ (labeling) $<10 \mathrm{~s}$, with protein and inhibitor both at $\left.1 \mu \mathrm{M}\right]$; the rate constant for the second-order reaction $\left(k_{2}\right)$ between acrylamide and cysteine $<0.1 \mathrm{M}^{-1} \mathrm{~s}^{-1}\left[t_{1 / 2}\right.$ (labeling) $>10^{7} \mathrm{~s}$, with protein and inhibitor both at $1 \mu \mathrm{M}$ ]. Although older modus operandi to achieve covalent inhibition, e.g., direct alkylation and mechanism-based inhibition exist, ligandable covalent binding has become a go-to strategy. ${ }^{1,2}$

It is clear that for ligandable designs, we need both a non-covalent binding site on the target protein matching the non-covalent moiety of the inhibitor, and a nearby nucleophilic residue to trap out the enzyme-inhibitor complex. Should there be no reactive residue, but the reversible portion can still bind the protein, the inhibitor will function reversibly. For such interactions, in patients and model organisms, upon inhibitor clearance (or removal of the compound from media in cultured cells), inhibition will diminish. However, for proteins that form a covalent bond to the bound inhibitor, inhibition is sustained until new protein is synthesized, regardless of clearance (Fig. 1). Thus, covalent appendages can help home in on specific targets of more promiscuous non-covalent binders, and raise the burden on targeted cells, while minimizing the impact of clearance. Several other benefits of covalent binders have also been proposed, including overcoming competitive ligands, and targeting "shallow binding sites". Work has justifiably focused on matching the ligandable interaction with the protein of interest (POI), and picking cysteines of convenience or genetic aberrations (e.g., oncogenic 
$\left.\operatorname{ras}^{\mathrm{G12C}}\right)^{3-5}$ as targets. This drive has led to a good deal of drugs approved in this millennium, that target cysteines including: afatinib ${ }^{6}$ and zanubrutinib ${ }^{7,8}$. Many similar molecules are in clinical trials and drives to identify novel ligandable interactions are common. Recently this strategy was extended to targeting $\mathrm{N}$-terminal amines ${ }^{9}$, through approval of Voxelotor to treat sickle cell disease. ${ }^{10,11}$ Herein we will discuss an alternative design strategy harnessing a ligandable interaction to orchestrate a selective privileged thiol-electrophile interaction. We will discuss why such an approach is conceptually different from the traditional viewpoint, and we will describe ways to predict mode of action, validate on target phenotypes, and data derived from such studies.

A.

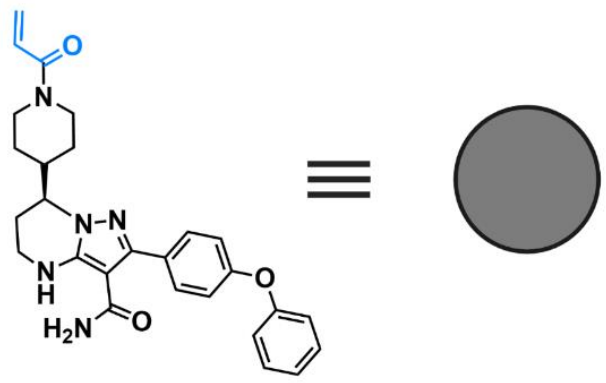

Zanubrutinib (2019)

B.

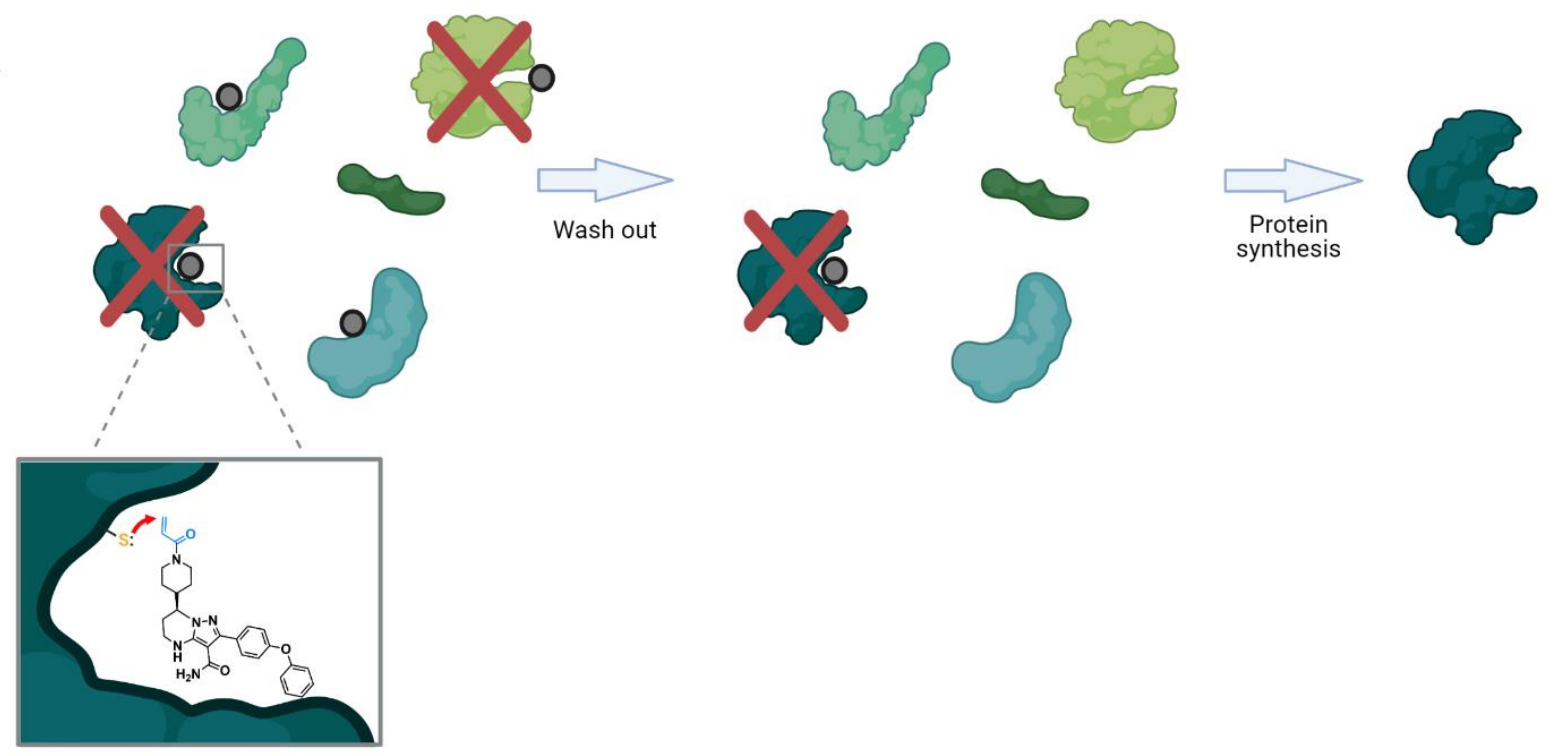

Figure 1. (A) Structure of Zanubrutinib, a covalent drug approved in 2019 for treatment of mantle cell lymphoma. The black ligand motif of the molecule allows binding to the adenosine-triphosphate binding pocket of its target, while the blue (Michael acceptor-based electrophilic motif) is covalently modified by nucleophilic residue from the protein. (B) General structure of a covalent inhibitor bound to its target. After binding, the Michael acceptor situated proximal to the nucleophilic residue, favors the formation of a covalent bond between the target protein and the drug molecule. This forms the irreversibly-bound ligand-protein complex that remains in vivo until new protein synthesis. However, if the reactive residue (Cys in this example) is not present, the inhibition will be reversible. 


\section{Privileged thiol-electrophile interactions}

In the 1950's Betty and Jim Millar ${ }^{12}$ established a strong link between exposure to reactive electrophiles and cancer. This link was extended to other disease etiologies, like drug-induced liver injury ${ }^{13}$ and neurodegeneration. ${ }^{14}$ So, when electrophiles were detected in cells and dietary samples, it was first thought that these molecules were unwanted and dangerous. Contrary to such expectations, the preponderance of data shows that life has harnessed naturally-produced electrophiles to change decision making and meaningfully modulate proteins controlling important signaling pathways: low concentrations of native electrophile externally added to cell culture media promote growth and aid recovery and survival under stress. However, at higher concentrations, these electrophiles behave as billed and are toxic. This hormesis indicates that electrophiles have multiple biological behaviors, and that they are uniquely context-dependent. ${ }^{15}$

Electrophiles created in an unstressed cell are degraded rapidly ${ }^{16}$, constricting their diffusion distance likely to much shorter than the length of a cell, perhaps even shorter than the diameters of some organelles. ${ }^{15}$ However, to signal, an endogenous electrophile must covalently engage a target, a timedependent process. For our purposes, we will consider only proteins, although such arguments could apply to other macromolecules. Thus, a sufficient amount of labeled protein must arise prior to clearance of the electrophile for phenotypes to be observed. Assuming that one binding event leads to inhibition of one target protein molecule, then typically $\sim 50 \%$ occupancy is minimally necessary to trigger a phenotype. Such a scenario mimics a haploid state. For numerous, although likely not a majority of genes, such a state can elicit a phenotypic change relative to wild-type. However, for numerous proteins, including enzymes in important pathways, $>>50 \%$ activity reduction is required to elicit a phenotypic change relative to wild-type. ${ }^{17}$ Such criteria set a high burden in terms of necessary occupancy, especially for electrophiles, to achieve, given that electrophiles are short-lived and their concentrations in healthy cells must be limited as they are toxic. 
High occupancy of an electrophile on a specific protein may be engendered by coordinating localized electrophile release with localization of the target protein (Fig. 2A). This choreography stimulates the intended bimolecular reaction over others that may occur if the electrophile were in the bulk of the cell. To analyze this process, we must consider concentration of electrophile and protein, and the second-order rate of reaction $\left(k_{2}\right)$. Owing to limitations/concerns underlying probes, and an inability to measure concentrations locally, we do not know particularly well local cellular concentration of even the most well-studied endogenous lipid-derived electrophile, 4-hydroxynonenal (HNE), or how long elevated HNE levels are sustainable. ${ }^{15,16}$ However, HNE concentrations can unlikely exceed low millimolar levels, a concentration that when administered externally, is toxic; such levels are likely not sustainable. Although there are exceptions, most protein concentrations are not significantly more than micromolar in cells ${ }^{18,19}$, with many being lower. However, subcellular or localized protein concentrations are less established. The $k_{2}$ for HNE reacting with cysteine is $\sim 1 \mathrm{M}^{-1} \mathrm{~s}^{-1}$. A similar $k_{2}$ has been reported for numerous protein cysteines engaging with HNE in vitro. At $1 \mathrm{mM} \mathrm{HNE}$, the half-life of labeling of a by-stander cysteine under pseudo first-order kinetics is 12 minutes; at $0.1 \mathrm{mM} \mathrm{HNE}$, $t_{1 / 2}=120$ minutes. Thus, the localized model results in relatively slow labeling even with optimistic HNE concentrations. This model also does not also necessarily lead to selective labeling of the target protein. This is because there are almost certainly many cysteines present in any given cellular region, many of which have $k_{2}(\mathrm{HNE})=1 \mathrm{M}^{-1} \mathrm{~s}^{-1}$. This concern may be assuaged since the vast majority of electrophile-labeling events may not affect protein-activity/function (although few have been adequately studied). However, as electrophile labeling is ostensibly irreversible ${ }^{20}$, and it can introduce reactive groups, e.g., aldehydes into the protein, the reasoning above seems an unsatisfying defense (Fig. 2B).

Thus, other mechanisms likely help promote electrophile-labeling selectivity and allow signaling to occur at low HNE concentrations. In our opinion the most likely mechanisms involve elevating $k_{2}$ (for $\mathrm{HNE}$, this could be up to $\sim 10^{8}$ fold, the ratio of the diffusion-limited $k_{2}$ for protein labeling: $k_{2}$ (cysteine)), and/or mechanism of activity modulation. ${ }^{15,21}$ For the former, by elevating $k_{2}$, a high 
occupancy can be achieved in a short time, prior to off-target labeling, regardless of the presence of other proteins. For the latter, the more impact a single electrophile labeling event has on the bulk contingent of the target (or other) protein(s), the less percentage labeling is needed. Note that lowering required threshold occupancy is particularly important for second-order reactions, where driving high occupancy becomes increasingly longer duration relative to a pseudo-first order process. To give optimal outputs, the mechanisms above could function in tandem. Electrophile-sensor proteins functioning through dominant pathways and displaying kinetically-favorable modification rates are termed privileged first responders (PFRs). ${ }^{16}$

A.

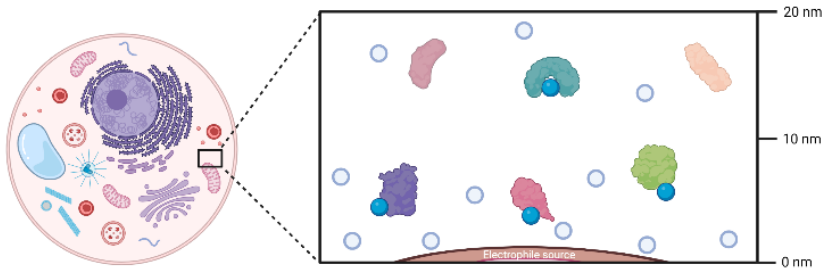

Electrophile

Engaged electrophile

B.

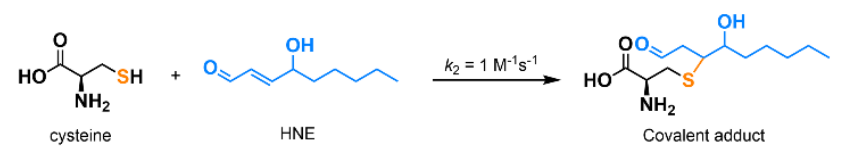

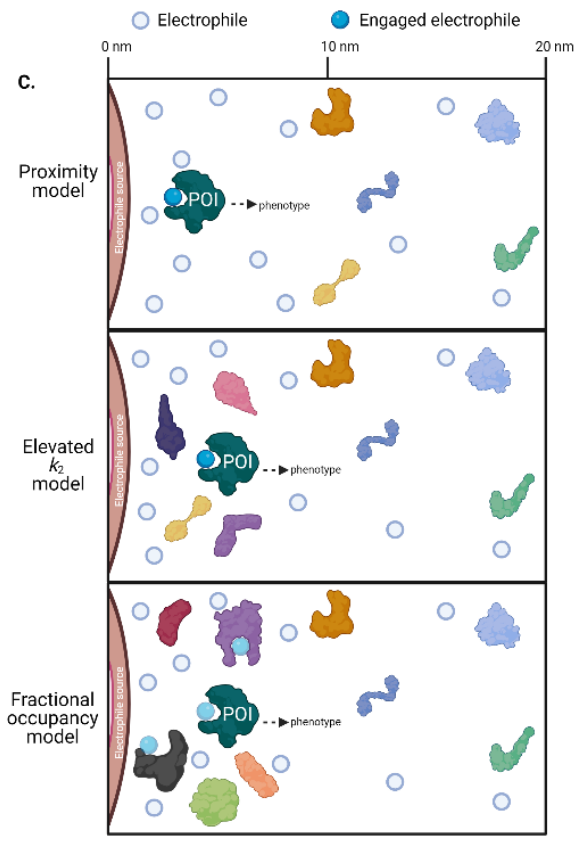

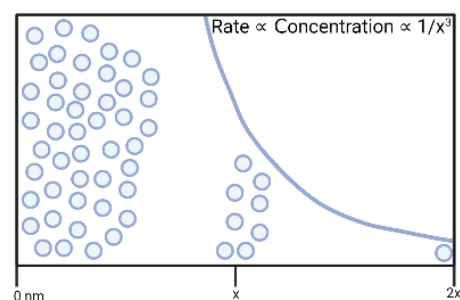

Figure 2. (A) Because of their high reactivity, electrophiles covalently engage with nearby targets, and covalent bond formation occurs in a time-dependent manner. The scheme shown exemplifies a case where more than $50 \%$ of protein labeling (by the electrophile) is required to elicit a phenotypic change. Should the protein have enzymatic properties, this functional output may be reflected in modulation of enzymatic activity. (B) Native lipid-derived electrophilic metabolite, HNE, reacts with free/non-protein-bound cysteine to form an aldehyde-containing product that can undergo subsequent nucleophilic substitution. (C) Top three panels: there are three possible models to explain how low concentration of HNE (generated from a point source, e.g., from an organellar lipid membrane during lipid peroxidation) can selectively modify a protein target and thus promote signaling. X-axis designates distance from the point source. The proximity model (top panel) is relatively slow and unselective at labeling, given that many proteins have at least one cysteine residue. On the other hand, the elevated $k_{2}$ model (middle) allows high occupancy to be achieved in a short time and/or greatly modulates bulk activity in a more impactful manner than the fractional occupancy model (bottom). Lowest panel: the rate of labeling (axis in Y direction as drawn) is proportional to the concentration of the electrophile that decreases as a function of the spherical volume of the cell (axis in $\mathrm{X}$ direction as drawn). POI, protein of interest. 


\section{The privileged, few?}

From the discussion above, we can predict that since cysteine is present at least once in most proteins, native reactive electrophiles like HNE, or related small-molecule covalent drugs, will label multiple proteins under prolonged bolus-dosing conditions. Such promiscuity is most easily shown by fluorescence imaging of SDS-PAGE analysis of lysates of cells which had been treated with a clickable variant of native electrophiles such as $\mathrm{HNE}^{22,23}$, fumarate ${ }^{24}$, or itaconate ${ }^{25}$, or related Michaelacceptor-based drug, dimethyl fumarate ${ }^{26}$ prior to lysis, and were then treated with a clickable dye. The number of bands appearing in the soluble proteome is impressive. However, such gel-based analysis does not show occupancy of the targets, so many of the detected labeling events may not be significant. as touched on above, for many targets, covalent ligandability may also not well lead to functional output. Similar multi-target- and time-dependent-labeling events are observed in aged tissues using HNE-antibodies ${ }^{27}$, although HNE-antibodies should be treated with caution ${ }^{28}$ as they are likely not particularly specific and correct control experiments cannot easily be used to validate them.

Data from several laboratories brought to light that electrophiles are not as promiscuous as may have been expected. Iso-TOP-ABPP demonstrated that significant (>75\%) labeling of a handful out of $\sim 1000$ cysteines occurs upon treatment of lysates with relatively high HNE concentrations. ${ }^{29}$ Extrapolating this ratio over the whole cysteome predicts hundreds to thousands of HNE-sensitive cysteines. This ballpark analysis agrees with more proteome-wide data from dosing/mass-spectrometry regimens that indicated there are hundreds $\mathrm{s}^{30}$ of HNE-sensitive proteins $\mathrm{s}^{31-33}$. Experiments using bolus dosing of cells with alkyne- or azide-modified electrophiles, biotin-Click, and streptavidin enrichment reach similar numbers. Labeling of some of these sensors, for related electrophiles at least, appears to be stimulated by oxidative stress. ${ }^{34}$ In situ derivation of a chemically-diverse mixture of lipid-derived electrophilic species specifically in the mitochondria, showed enrichment principally of mitochondrial proteins, giving weight to location being critical to determine lipid-modification processes. 
In the few cases where different chemotypes have been compared, it has transpired that labeling is unexpectedly chemoselective. For instance, iso-TOP-ABPP measurements showed little overlap of targets between HNE and a prostaglandin-derived electrophile. Other examples of this phenomenon exist, although PFRs are not always chemoselective, especially for proteins with multiple sensor cysteines.

\section{Limitations thus far}

Many of the above methods were conducted in lysates and/or deploy procedures where dose and timing of electrophile is exaggerated relative to what likely occurs in cells. Such conditions likely artificially elevate chances of observing a labeling event because mass spectrometry is particularly sensitive, and HNE labels targets not only in a dose but also time-dependent manner. ${ }^{16}$ Furthermore, as the methods above cannot absolutely and/or directly quantitate occupancy, we see that sensors with moderate reactivity, or cysteines sensitive to the environment established under experimental conditions, may have been identified. Conversely, as reactive cysteines are likely sensitive to their environment, the procedures used could readily mask reactive cysteines. As most methods also use an enrichment protocol but with differing elution/detection strategies, it is hard to know what selection occurs due to different protocols of enrichment, clearance, and elution.

\section{Identifying phenotypically-relevant sensors}

It is also clear that the above experiments are blind to dominant signaling behavior, and ultimately give limited insight into links between ligand binding and biological output in general. Furthermore, as experiments seeking to gain quantitative insight by ranking efficiency of labeling of proteins or sites rank based on total occupancy by an electrophile (i.e., ignoring locale, associating factors, etc.); context-specific sensors, which can achieve fractional occupancy rapidly but fail to saturate at their 
HNEylated site(s), are often below the MS-cut-off threshold. Thus, identifying nuanced aspects that are likely to exist if electrophile signaling functions as predicted are outside the grasp of most methods.

Once targets of specific signaling molecules are assigned, typically their role in biological signaling can be addressed to fill in some of the baps outlined above. For instance, should labeling of a POI be necessary for a specific phenotype, its knockout/knockdown/loss of function should diminish the associated phenotype observed on electrophile treatment. This approach has been applied to numerous traditional biological problems, including a host of screens. A plethora of published data demonstrate that this stratagem has not worked well with reactive electrophiles. There are numerous potential reasons for lack of success, including: detection methods not identifying the correct sensors; the manifold pathways and target proteins modulated by these reactive small molecules synergize to a "compound" phenotype with no single target necessary or sufficient for cellular response; there are multiple sensors, of which the majority are sufficient, but not necessary to cause signaling (Fig. 3). The first reason likely contributes to the issues in this area, as discussed above. The second reason is essentially untestable in the form it is presented. The third reason is mutually exclusive to the second and is further testable provided we can form precisely defined electrophile-modified states and interrogate them in an unperturbed cell. Should labeling of an individual POI be sufficient to trigger labeling, the state so formed, will necessarily change signaling. 

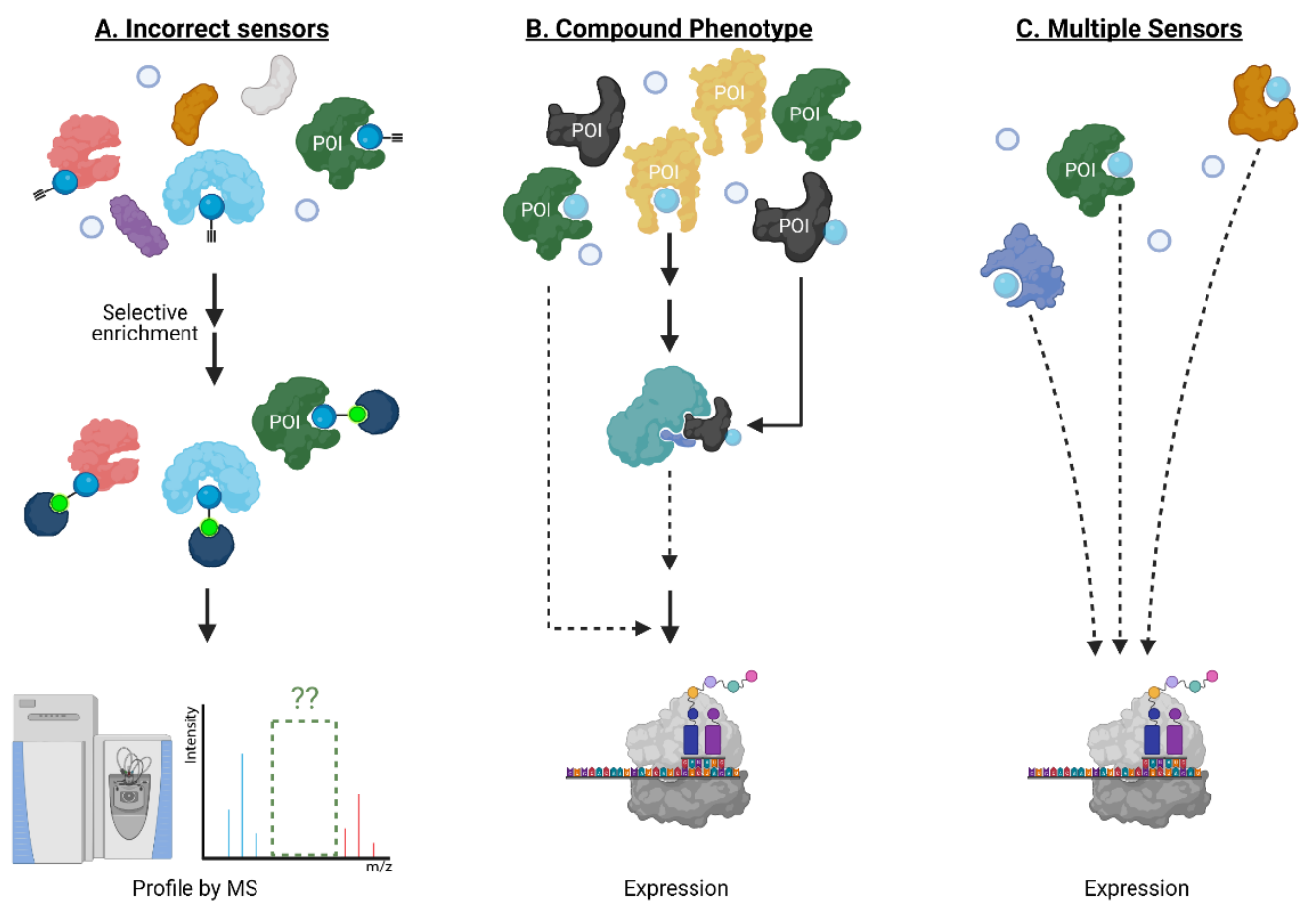

Figure 3. Tradition knockdown/knockout/loss-of-function screens of a protein of interest (POI) labeled by electrophiles have not worked well for the following reasons: (A) the kinetically-privileged electrophile-sensor protein has not been correctly identified by state-of-the-art methods which remain limited in terms of, for instance, reliance on indirect readout using proxy electrophiles, limited ability to identify targets with low ligand occupancy, and lack of spatiotemporal resolution in intact living systems; (B) multiple pathways and targets must be modified to achieve the observed overall phenotype, such as protein translation for instance; and (C) multiple sensors are present and their labeling/site occupancy is sufficient, but modification is not necessary to cause signaling. Effect on translation is shown just as an example but many biological processes have been implicated to be affected by electrophilic protein modificaitons.

\section{REX technologies a new way to study electrophile-protein engagement}

To investigate if electrophile engagement by specific sensors be sufficient to trigger signaling, we developed T-REX. ${ }^{35}$ This method labels a specific, preordained protein with a specific electrophile of choice, providing that protein be a kinetically privileged sensor of the specific electrophile in question. This constraint is applied because the native electrophile is offered to the POI pseudointramolecularly. Then, the POI can engage with the electrophile, or the POI can leave the electrophile to diffuse away, irreversibly; under T-REX conditions, second order labeling of the POI by the released electrophile is minimal. The amount of electrophile released to the POI, and the amount of electrophile on the POI, are quantifiable. Thus, T-REX can quantitatively assess POI electrophile occupancy. The majority of proteins are not labelled under T-REX. Furthermore, proteins that are 
labelled by T-REX in cells show rapid in vitro $k_{2}(\mathrm{HNE}) .{ }^{15,35,36}$ These observations are consistent with TREX labeling only the best electrophile sensors. Finally, HNE released to the bulk proteome under TREX does not affect numerous HNE-sensitive signaling processes. Thus, phenotypes measured under T-REX are due to labeling of the POI.

For several HNE-sensitive proteins, T-REX delivery efficiency (DE) between 15 and 50\% triggers signaling phenotypes. ${ }^{22,23,36-42}$ In several instances the phenotypes stimulated are similar in magnitude to those observed under bolus dosing, or when an inhibitor is used to fully shut down POI activity. Thus, specific electrophile labeling of single proteins is sufficient to trigger signaling. Signaling changes measured are significantly greater than predicted based on measured occupancy. This result demonstrates that dominant signaling modes occur in redox sensing, and as this has now been shown for several different proteins/pathways (NF-KB ${ }^{35,43}$, PI3K/Akt ${ }^{35,41,44}$, DNA-damage ${ }^{43}$, and antioxidant response ${ }^{22,23,35,37-39,42}$ signaling), we cautiously postulate that such behaviors are not uncommon. Such results reconcile some indirect data that had shown that the occupancy on postulated electrophile sensors necessary to trigger signaling was relatively small. ${ }^{15,36,37,42}$

Although T-REX-mediated electrophile release is chemospecific, $\sim 10$ different electrophiles so far, and likely many more, are compatible with this method. T-REX thus can perform unbiased (i.e. independent of permeation, metabolism, distribution, etc.) structure activity relationships (SAR) to identify an optimal match between electrophile and target protein in terms of both kinetics and phenotype/dominance in cells and organisms. These considerations all together led us to propose that $\mathrm{PFRs}^{45}$ are ideal for covalent drug design and that T-REX is an ideal means to predict drug phenotypes and inform on covalent drug design.

\section{Translating T-REX data}


In our translational studies, we investigated the isoform-specific inhibition of Akt3 kinase by HNEderived electrophiles. Akt is upregulated in numerous cancers, and is a downstream effector of PIK3, for which an inhibitor, alpelisib, was recently approved to treat breast cancer. ${ }^{46}$ However, Akt has 3 different isoforms, encoded at differing genetic loci in humans and linked to different cancers. Thus, there is a growing need for Akt-isoform specific inhibitors. Some success has been reached with partly Akt1-selective inhibitors, with drugs such as MK2206 entering phase II clinical trials. ${ }^{47-49}$ However, there is generally a pressing need for drug strategies targeting specific isoforms or specific alleles that is only partially met. Some success has been achieved in this area, such as the approval of Ayvakit to treat patients with a PDGFRA exon 18 mutation, although much work is left to be done..$^{50}$ In the case of Akt isoforms, Akt3 is a particularly difficult to target, despite being implicated in triple negative breast cancer etiology. Intriguingly, when comparing electrophile sensitivity of Akt isoforms by T-REX, Akt3 emerged as the most HNE-reactive isoform. Electrophile labeling occurs on a cysteine unique to Akt3, C119. ${ }^{41} \mathrm{C} 119$ lies in an isoform-divergent linker region separating the two principal domains of Akt, the plekstrin homology domain and the kinase domain. Critically, Akt3(C119) electrophile engagement leads to dominant negative (DN) inhibition of Akt activity. Akt2 is labelled by HNE, although this occurs approximately four-fold less efficiently than for Akt3 and is not DN. Akt1 is not targeted by electrophiles.

We chose to derive an inhibitor from the Akt1-semi-selective inhibitor, MK2206, that targets Akt3 100-fold less effectively. This inhibitor binds to an allosteric site common to all Akts. This choice was made because C119 is removed from the active site, where many Akt-targeted molecules bind: the MK2206-binding site is closer to the flexible linker region. We also wanted to pit the two segments of the chimeric inhibitor together, so it was good that the reversible interaction favors Akt1, and the covalent portion favors Akt3. The resulting molecule, MK-HNE and its second-generation inhibitor, MK-FNE, were selective inhibitors of Akt3, showing no labeling of Akt1, and weaker labeling of Akt2 ( 3.5-fold <Akt3). ${ }^{44}$ MK-FNE enacted irreversible inhibition of Akt3 and Akt2. For Akt3, inhibition was DN: when a mutant Akt3 that cannot bind MK-HNE [Akt3(C119S)] was expressed 1:1 with wild type 
Akt3, 100\% inhibition was retained, even post drug washout. Repeating this experiment with Akt2, significant regain of activity was observed. MK-FNE emerged to be more selectively cell line toxic than MK2206, and it further showed positive synergy with Akt3 (and not Akt1/2) knockdown in sensitive lines. MK2206 showed positive synergy with Akt1 (and not Akt2/3) knockdown. MK-FNE emerged to be more potent and showed less side effects than MK2206 in mouse xenograft models of breast cancer.

T-REX studies of Akt3 labeling had shown that Akt(T305) phosphorylation was unaffected by HNEylation. Conversely, MK-2206 treatment of cells reduced Akt3(T305) phosphorylation. When we examined Akt3(T305) phosphorylation after Mk-FNE treatment, we saw no change, despite Akt3 inhibition being significant. Thus, the mode of action of MK-FNE is dominated by electrophile-protein engagement rather than MK-2206 engagement.

\section{Going forward}

T-REX, therefore, can inform on covalent drug design, and further can predict bespoke traits of the electrophile-protein interaction, while also informing on target profiles. These points are encouraging. It is important to consider what aspects of T-REX assisted in making such data possible and further what aspects of this strategy could be improved or could be better leveraged in the future.

Akt3's HNE sensitivity was discovered using a medium-throughput T-REX screen. This is an arduous process that is likely not possible on the whole proteome and further compounds inherent limitations of T-REX. We have now established G-REX ${ }^{51,52}$, a method for unbiased profiling of electrophilesensitive proteins. Hits from G-REX will be fed into our pipeline later. However, generally, any electrophile panning program can be used to generate candidate PFRs for study by T-REX, and then drug design as we outline. We indeed encourage laboratories claiming to have discovered HNE- 
sensitive proteins to interrogate HNE-labeling and phenotypes induced by unambiguous labeling prior to making such claims.

The ramifications of Akt3-HNE labeling were evaluated rigorously in cell culture, relative to a defunct mutant and relative to an isoform that did not sense electrophiles. These experiments were also repeated in zebrafish, where similar labeling and signaling were observed, in an isoform-specific manner. Showing robustness of phenotypes and transmission is certainly important prior to going forward to drug discovery. However, such experiments would ideally be carried out in the model organism of choice for drug testing, which in our case, as for many preclinical studies, was mice. Unfortunately, mice are not applicable to T-REX as our photouncaging methodology is not compatible with whole mice. T-REX may, be applicable to specific tissues, or organs, and circulating cells, like blood cells. Time will tell how important advanced T-REX testing be for triaging hybrid molecules.

The effects of T-REX and MK-FNE were studied at the level of Akt activity, Akt3 phosphorylation, various immediate downstream signaling processes, and occupancy. These agreed well and together provide a very convincing picture that HNE is the determinant of MK-FNE activity. However, we did not develop a detailed picture of the changes in transcriptome, chromatin, etc that could have been rigorously compared with MK-2206 treatment, and the hybrid molecule. Leveraging deep sequencing methods would allow establishment of more quantitative signatures arising due to target engagement. Comparison of signatures caused upon T-REX to those from drug candidates could also allow testing of on-target behavior in a more detailed manner. Our early experiments in cells and fish, as yet unpublished, indicate the T-REX protocol is applicable to RNA-seq experiments, although these are best performed against a sensor cysteine mutant control. However, given T-REX can only achieve low occupancy on the target, comparisons with saturating concentrations of a drug, may prove to be difficult to analyze.

Typically, the root cause of inhibition in classic inhibitor designs is derived from the non-covalent interaction; in most cases it is unknown how covalent electrophile labeling of the cysteine affects 
activity (although in some cases oxidation of the targeted residue stimulates activity ${ }^{53}$ ). What is clear is that targeting PFRs is accessible to scenarios in which non-covalent interaction with the target is not inhibitory. This is important because there has been an increase in assays screening for protein binders, yielding a wealth of binders reported in the literature. It will be important to test how effective such covalent inhibitors can be in the future.

The DE Akt3 was approximately $20 \%$, and inhibition was DN. ${ }^{41}$ This DE is not the highest we have reported. However, DE(Akt3) is higher than several proteins we have studied, such as RNR and HuR. For HuR, there was no change in activity upon electrophile labeling, even though similar DE stimulated Ube2V1 to promote Ube2N activity in cells. Such an outcome for HuR could arise due to: DE being too low; lack of/insufficiency of dominance of signaling; because the HuR activity measured was not affected by electrophile labeling; or possibly because of experiment-specific variations such as protein expression/expression of associated factors; etc. Whatever it be, as the phenotype engendered by the electrophilic moiety becomes less impactful, T-REX experiments may be less able to predict phenotypes. In such scenarios, it would be logical to change the electrophile released in a bid to improve signaling properties, which T-REX offers a means to achieve. Regardless of the effects of such SAR, there remain benefits in terms of targeting kinetically privileged cysteines, as they are inherently reactive, a property that will transpire to good inhibitor kinetics, although it is unknown how necessary such properties are to derive a ligandable covalent drug and many covalent drugs have been developed without ostensibly targeting PFRs. Investigating the electrophile sensitivity and privileged signaling properties of cysteines targeted by approved drugs using a method like T-REX would also therefore be insightful.

The discussion above, nonetheless, shows it is important that threshold parameters be established for translating data from T-REX to a ligandable inhibitor. Although precise thresholds may not be realizable (due to mechanistic changes, locale-specific sensing effects, etc.), having a series of experimentally-validated criteria derived from numerous electrophile scaffolds and different PFRs 
could certainly be useful to triage hits from screening efforts. We now possess enough proteins to perform such experiments, although not all of these have accommodating reversible ligands. Akt, fortunately is the target of numerous drugs, and $\mathrm{HNE}(\mathrm{FNE})$-derivatization of these molecules could readily inform on how Akt isoform affinity/selectivity affects drug efficacy and isoform selectivity. Similar chemical structural changes carried out across a series of ligandable interactions could inform other variables, such as linker preferences, although these are more likely to be idiosyncratic, as has been found for many chimeric drugs.

Another clear issue going forward is how well is the molecular design able to suppress HNE's pleotropic tendencies? We know that kinetically we have reduced the inherent reactivity of HNE by several orders of magnitude by converting the aldehyde to an amide. The $k_{\text {inact }} / \mathrm{k}_{\mathrm{i}}$ for Akt3/MK-FNE is $\sim 10^{4} \mathrm{M}^{-}$ ${ }^{1} \mathrm{~s}^{-1}$, which is $\sim 6$ orders of magnitude faster than the standard rate of a $\beta$-substituted enamide with a cysteine. ${ }^{15,41,44}$ This is close to the maximum rate enhancement possible for HNE (108-fold) with a protein of interest. The $k_{\text {inact }} / k_{i}$ is also faster than any second order rate reported for reaction between HNE and a target protein, although these rates are poorly studied in general, and we may never know all the best sensors. For MK-FNE we evaluated off-target effects both in mice (these were less than MK-2206,) and positive synergy of MK-FNE was observed only with Akt3 knockdown, whereas positive synergy was only observed with Akt1 for MK2206 in cell culture. As our T-REX studies had shown that C119 within Akt3 was the cysteine attacked by MK-HNE/FNE we were also able to perform some experiments with Akt3(C119S), which is resistant to covalent targeting by MK-FNE/HNE. Prior to advanced testing, performing experiments on xenografts of cells expressing Akt3(C119S) at endogenous levels would be an important step.

A related issue is, is HNE a good handle for drug design? It is true that the hydrophobic portion of HNE is not present in many drugs, although similar functions are present in approved drugs such as fulvestrant ${ }^{54}$, symmetrel ${ }^{55}$ and flumadine ${ }^{56}$, and tamoxifen ${ }^{57}$ Click staining of xenografts treated with MK-FNE also showed significant permeation into the tumor post oral gavage. However, how 
hydrophobic tails affect subcellular distribution is another issue we have yet to address. Issues with off-target effects could be different for molecules like MK-HNE/FNE and fulvestrant.

\section{Conclusion}

We therefore believe that we have provided good evidence that biological activity can be modulated in a DN fashion through specific covalent engagement with a specific electrophile. If this engagement be ushered through a ligandable interaction, even if that ligandable interaction should lead to measurable effects on the target protein, the covalent handle can exert its effects in a manner predictable based on data derived from studying protein-specific electrophile labeling. Using T-REX or other strategies that may arise to model protein specific electrophile engagement we can hone our resources to focus on the most responsive target proteins, and interrogate off-target effects in ways that were not previously possible. 


\section{References}

1 P. A. Schwartz, P. Kuzmic, J. Solowiej, S. Bergqvist, B. Bolanos, C. Almaden, A. Nagata, K. Ryan, J. Feng, D. Dalvie, J. C. Kath, M. Xu, R. Wani and B. W. Murray, Proceedings of the National Academy of Sciences, 2014, 111, 173-178.

M. J. C. Long, A. Kulkarni and Y. Aye, ChemBioChem, 2021, cbic.202100051.

J. M. Ostrem, U. Peters, M. L. Sos, J. A. Wells and K. M. Shokat, Nature, 2013, 503, 548-551.

R. S. Goody, M. P. Müller and D. Rauh, Cell Chemical Biology, 2019, 26, 1338-1348.

D. Y. Yoo, A. D. Hauser, S. T. Joy, D. Bar-Sagi and P. S. Arora, ACS Chem. Biol., 2020, 15, 1604-1612.

R. T. Dungo and G. M. Keating, Drugs, 2013, 73, 1503-1515.

Y. Guo, Y. Liu, N. Hu, D. Yu, C. Zhou, G. Shi, B. Zhang, M. Wei, J. Liu, L. Luo, Z. Tang, H. Song, Y. Guo, X. Liu, D. Su, S. Zhang, X. Song, X. Zhou, Y. Hong, S. Chen, Z. Cheng, S. Young, Q. Wei, H. Wang, Q. Wang, L. Lv, F. Wang, H. Xu, H. Sun, H. Xing, N. Li, W. Zhang, Z. Wang, G. Liu, Z. Sun, D. Zhou, W. Li, L. Liu, L. Wang and Z. Wang, J. Med. Chem., 2019, 62, 7923-7940.

B. Metcalf, C. Chuang, K. Dufu, M. P. Patel, A. Silva-Garcia, C. Johnson, Q. Lu, J. R. Partridge, L. Patskovska, Y. Patskovsky, S. C. Almo, M. P. Jacobson, L. Hua, Q. Xu, S. L. Gwaltney, C. Yee, J. Harris, B. P. Morgan, J. James, D. Xu, A. Hutchaleelaha, K. Paulvannan, D. Oksenberg and Z. Li, ACS Med. Chem. Lett., 2017, 8, 321-326.

J. Han, S. L. Saraf and V. R. Gordeuk, Pharmacotherapy, 2020, 40, 525-534.

A. Hutchaleelaha, M. Patel, C. Washington, V. Siu, E. Allen, D. Oksenberg, D. D. Gretler, T. Mant and J. Lehrer-Graiwer, Br J Clin Pharmacol, 2019, 85, 1290-1302.

A. A. Taylor, Journal of Biological Chemistry, 2017, 292, 12008-12009.

K. Mitra, W. Kang, K. Tanis, S. Pacchione, M. Su, Z. Wang, K. Bleicher, T. Griffiths, G. Laws, M. Kuhls, A. Podtelezhnikov, J. Monroe, T. Johnson, D. Marsh, I. Knemeyer, Q. Chen, J. Lebron and F. Sistare, Drug Metabolism and Pharmacokinetics, 2019,34, S42.

S. Parvez, M. J. C. Long, J. R. Poganik and Y. Aye, Chem. Rev., 2018, 118, 8798-8888.

X. Liu, M. J. C. Long and Y. Aye, Trends in Biochemical Sciences, 2019, 44, 75-89.

W. F. Eanes, T. J. S. Merritt, J. M. Flowers, S. Kumagai, E. Sezgin and C.-T. Zhu, Proceedings of the National Academy of Sciences, 2006, 103, 19413-19418.

X. Fang, Y. Fu, M. J. C. Long, J. A. Haegele, E. J. Ge, S. Parvez and Y. Aye, J. Am. Chem. Soc., 2013, 135, $14496-14499$.

R. A. Kulkarni, D. W. Bak, D. Wei, S. E. Bergholtz, C. A. Briney, J. H. Shrimp, A. Alpsoy, A. L. Thorpe, A. E. Bavari, D. R. Crooks, M. Levy, L. Florens, M. P. Washburn, N. Frizzell, E. C. Dykhuizen, E. Weerapana, W. M. Linehan and J. L. Meier, Nat Chem Biol, 2019, 15, 391-400.

W. Qin, Y. Zhang, H. Tang, D. Liu, Y. Chen, Y. Liu and C. Wang, J. Am. Chem. Soc., 2020, 142, 10894-10898.

I. Kastrati, M. I. Siklos, E. L. Calderon-Gierszal, L. El-Shennawy, G. Georgieva, E. N. Thayer, G. R. J. Thatcher and J. Frasor, Journal of Biological Chemistry, 2016, 291, 3639-3647. 
33 B. K. Chacko, S. B. Wall, P. A. Kramer, S. Ravi, T. Mitchell, M. S. Johnson, L. Wilson, S. Barnes, A. Landar and V. M. Darley-Usmar, Redox Biology, 2016, 9, 57-66.

34 T. D. Cummins, A. N. Higdon, P. A. Kramer, B. K. Chacko, D. W. Riggs, J. K. Salabei, L. J. Dell'ltalia, J. Zhang, V. M. Darley-Usmar and B. G. Hill, Free Radical Biology and Medicine, 2013, 59, 56-68.

35 S. Parvez, M. J. C. Long, H.-Y. Lin, Y. Zhao, J. A. Haegele, V. N. Pham, D. K. Lee and Y. Aye, Nat Protoc, $2016,11,2328-2356$.

36 S. L. Surya, M. J. C. Long, D. A. Urul, Y. Zhao, E. J. Mercer, I. M. Elsaid, T. Evans and Y. Aye, ACS Chem. Biol., $2018,13,1824-1831$.

37 J. R. Poganik, A. K. Van Hall-Beauvais, M. J. C. Long, M. T. Disare, Y. Zhao and Y. Aye, HCA, , DOI:10.1002/hlca.202000041.

38 H.-Y. Lin, J. A. Haegele, M. T. Disare, Q. Lin and Y. Aye, J. Am. Chem. Soc., 2015, 137, $6232-6244$.

39 M. J. Long, H.-Y. Lin, S. Parvez, Y. Zhao, J. R. Poganik, P. Huang and Y. Aye, Cell Chemical Biology, 2017, 24, 944-957.e7.

40 M. J. C. Long, D. A. Urul, S. Chawla, H.-Y. Lin, Y. Zhao, J. A. Haegele, Y. Wang and Y. Aye, Biochemistry, 2018, 57, 216-220.

41 M. J. C. Long, S. Parvez, Y. Zhao, S. L. Surya, Y. Wang, S. Zhang and Y. Aye, Nat Chem Biol, $2017,13,333-338$.

42 J. R. Poganik, M. J. C. Long, M. T. Disare, X. Liu, S.-H. Chang, T. Hla and Y. Aye, The FASEB Journal, $2019,33,14636-14652$.

43 Y. Zhao, M. J. C. Long, Y. Wang, S. Zhang and Y. Aye, ACS Cent. Sci., 2018, 4, 246-259.

44 X. Liu, M. J. C. Long, B. D. Hopkins, C. Luo, L. Wang and Y. Aye, ACS Cent. Sci., 2020, 6, 892-902.

45 M. J. C. Long and Y. Aye, Cell Chemical Biology, 2017, 24, 787-800.

46 F. André, E. Ciruelos, G. Rubovszky, M. Campone, S. Loibl, H. S. Rugo, H. Iwata, P. Conte, I. A. Mayer, B. Kaufman, T. Yamashita, Y.S. Lu, K. Inoue, M. Takahashi, Z. Pápai, A.-S. Longin, D. Mills, C. Wilke, S. Hirawat and D. Juric, N Engl J Med, 2019, 380, $1929-1940$.

47 C. X. Ma, V. Suman, M. P. Goetz, D. Northfelt, M. E. Burkard, F. Ademuyiwa, M. Naughton, J. Margenthaler, R. Aft, R. Gray, A. Tevaarwerk, L. Wilke, T. Haddad, T. Moynihan, C. Loprinzi, T. Hieken, E. K. Barnell, Z. L. Skidmore, Y.-Y. Feng, K. Krysiak, J. Hoog, Z. Guo, L. Nehring, K. B. Wisinski, E. Mardis, I. S. Hagemann, K. Vii, S. Sanati, H. Al-Kateb, O. L. Griffith, M. Griffith, L. Doyle, C. Erlichman and M. J. Ellis, Clin Cancer Res, 2017, 23, 6823-6832.

48 A. P. Myers, P. A. Konstantinopoulos, W. T. Barry, W. Luo, R. R. Broaddus, V. Makker, R. Drapkin, J. Liu, A. Doyle, N. S. Horowitz, F. Meric-Bernstam, M. Birrer, C. Aghajanian, R. L. Coleman, G. B. Mills, L. C. Cantley, U. A. Matulonis and S. N. Westin, Int. J. Cancer, 2020, 147, $413-422$.

49 Y. Oki, M. Fanale, J. Romaguera, L. Fayad, N. Fowler, A. Copeland, F. Samaniego, L. W. Kwak, S. Neelapu, M. Wang, L. Feng and A. Younes, Br J Haematol, 2015, 171, 463-470.

$50 \quad$ U.S. Food and Drug Administration, 2020.

51 Y. Zhao, M. J. C. Long, Y. Wang, S. Zhang and Y. Aye, ACS Cent. Sci., 2018, 4, 246-259.

52 M. J. C. Long, C. Rogg and Y. Aye, Acc. Chem. Res., 2021, 54, 618-631.

53 C. E. Paulsen, T. H. Truong, F. J. Garcia, A. Homann, V. Gupta, S. E. Leonard and K. S. Carroll, Nat Chem Biol, 2012,8 , $57-64$.

54 J. F. R. Robertson and M. Harrison, Br J Cancer, 2004, 90, S7-S10.

55 V. Vernier, Toxicology and Applied Pharmacology, 1969, 15, 642-665.

56 A. M. Wallbank, Experimental Biology and Medicine, 1969, 131, 1025-1027.

57 M. Clemons, S. Danson and A. Howell, Cancer Treatment Reviews, 2002, 28, 165-180. 